\title{
Wogitare
}

\section{VIOLÊNCIA CONJUGAL E PRÁTICA ASSISTENCIAL POR NÍVEIS DE ATENÇÃO À SAÚDE: DISCURSO DE ENFERMEIRAS}

\author{
Anderson Reis de Sousa ${ }^{1}$ (i) \\ Fernanda Matheus Estrela² (i) \\ Andrey Ferreira da Silva ${ }^{1}$ (1) \\ Júlia Renata Fernandes de Magalhães ${ }^{1}$ (1) \\ Milena Arão da Silva Oliveira ${ }^{1}$ (1) \\ Adriana Karlla Neves da Silva Loureiro ${ }^{3}$ (1) \\ Valquíria Rodrigues Gomes ${ }^{4}$
}

\section{RESUMO}

Objetivo: descrever a prática assistencial da enfermeira frente aos casos de violência conjugal nos três níveis de atenção à saúde.

Método: estudo qualitativo realizado com 47 enfermeiras atuantes em uma grande cidade do Nordeste brasileiro. Os dados foram coletados entre setembro e novembro de 2018, sistematizados através do software NVIVO11®, e analisados por meio do Discurso do Sujeito Coletivo.

Resultados: a pesquisa despontou que, na identificação dos casos de violência, há necessidade de investigação do cotidiano conjugal e estabelecimento de vínculo com a usuária. No que tange aos encaminhamentos, é necessária articulação com outros profissionais.

Considerações finais: a pesquisa oferece subsídios para nortear o cuidado às mulheres em situação de violência conjugal nos três níveis de atenção.

DESCRITORES: Violência Doméstica; Níveis de Atenção à Saúde; Enfermagem; Saúde Pública; Saúde da Mulher.

\section{VIOLENCIA CONYUGAL Y PRÁCTICA ASISTENCIAL POR NIVELES DE ATENCIÓN SANITARIA: DISCURSO DE LAS ENFERMERAS}

\section{RESUMEN:}

Objetivo: describir la práctica asistencial de la enfermera ante los casos de violencia conyugal en los tres niveles de atención sanitaria. Método: estudio cualitativo realizado con 47 enfermeras que trabajan en una gran ciudad del Nordeste brasileño. Los datos fueron recolectados entre septiembre y noviembre de 2018, sistematizados a través del software NVIVO11®, y analizados a través del Discurso del Sujeto Colectivo. Resultados: la investigación reveló que, en la identificación de los casos de violencia, es necesario investigar la vida conyugal cotidiana y establecer un vínculo con la usuaria. En lo que respecta a los encaminamientos, es necesaria una articulación con otros profesionales. Consideraciones finales: la investigación ofrece subsidios para orientar la atención a las mujeres en situación de violência conyugal en los tres niveles de atención.

DESCRIPTORES: Violencia Doméstica; Niveles de Atención de Salud; Enfermería; Salud Pública; Salud de la Mujer.

${ }^{1}$ Universidade Federal da Bahia. Salvador, BA, Brasil.

${ }^{2}$ Universidade Estadual de Feira de Santana. Feira de Santana, BA, Brasil.

${ }^{3}$ Prefeitura Municipal de Ananindeua. Ananindeua, PA, Brasil.

^Universidade do Estado do Pará. Belém, PA, Brasil. 
A violência conjugal é um problema global que repercute na vida e saúde dos envolvidos e, deste modo, demanda um cuidado holístico em todos os níveis de atenção. Apesar da relevância da atuação do campo da saúde para a prevenção e enfrentamento desse fenômeno, nota-se a necessidade de maior preparo profissional para a abordagem dos casos, desde a sua identificação até a intervenção por meio da articulação intersetorial.

A Organização Mundial da Saúde (OMS) define três níveis de atenção à saúde, os quais se diferenciam pelo grau de complexidade. O nível primário compreende as unidades básicas responsáveis, sobretudo, pela prevenção de doenças e promoção da saúde; o secundário integra as unidades de pronto atendimento (UPA) e especialidades médicas com fins na estabilização de quadros clínicos e atendimento às especificidades orgânicas; e o nível terciário composto pelos grandes hospitais, com a finalidade de manutenção da vida ${ }^{(1)}$. Compreender o funcionamento, a finalidade e a inserção de cada unidade na rede de atenção pode fundamentar o trabalho da enfermagem e contribuir para uma prática mais efetiva diante da violência, considerando a sua ampla magnitude no contexto atual.

Revelando a expressividade desse fenômeno, estudo aponta que, em todo o mundo, cerca de uma a cada três mulheres já foi violentada por seu parceiro íntimo ${ }^{(2)}$. No contexto brasileiro, mais de 16 milhões de mulheres foram vítimas de violência somente no ano de $2018^{(3)}$. O conceito da violência doméstica, no qual se insere a violência conjugal, refere-se a qualquer forma de agressão física, sexual, psicológica, moral ou patrimonial praticada em ambiente doméstico, familiar ou em qualquer outro lugar, desde que baseada no gênero, conforme definição da Lei 11.340/06, conhecida como Lei Maria da Penha(4).

Esse cenário reverbera em adoecimento físico e mental, com a manifestação de lesões de pele, fraturas, lacerações, infecções sexualmente transmissíveis, além de doenças psicossomáticas e tentativas de autoextermínio, as quais levam as mulheres a procurarem os serviços de saúde em todos os níveis de atenção(5). Calcula-se que mais de 40 mil brasileiras em situação de violência conjugal adentrem o Serviço Único de Saúde (SUS), o que representa um custo superior a cinco milhões de reais ${ }^{(6)}$. Há ainda de se considerar as repercussões vivenciadas pelos homens e filhos que, por sua vez, também demandam atenção dos serviços de saúde.

Nesse sentido, é importante que os profissionais de saúde, atuantes nos mais variados níveis de complexidade, estejam aptos a reconhecer as situações de violência e a prestar o atendimento adequado. Ressalta-se, no entanto, que diversos estudos têm apontado as limitações apresentadas no cotidiano de trabalho pelos profissionais, tanto no reconhecimento quanto nas intervenções diante dos casos, o que pode estar relacionado à fragilidade na abordagem da temática durante a graduação e nos espaços de educação continuada ${ }^{(7-8)}$.

Considerando a violência como um fenômeno complexo, faz-se necessário compreender a prática assistencial pautada na integralidade, sendo esta uma diretriz do SUS, permitindo a identificação das mulheres em situação de violência enquanto sujeito histórico, social, político, considerando a sociedade em que vive, seu contexto familiar e suas particularidades ${ }^{(9)}$. Dessa forma, urge que seja utilizado o princípio da integralidade como eixo analítico nas práticas de saúde voltadas a esse público nos três níveis de atenção.

O compartilhamento de vivências e práticas realizadas por profissionais de saúde frente à violência contra a mulher pode subsidiar a reflexão acerca da maneira que o cuidado vem sendo prestado e até mesmo auxiliar no processo de capacitação profissional. Esse estudo então objetivou descrever a prática assistencial da enfermeira frente aos casos de violência conjugal nos três níveis de atenção à saúde. 
Trata-se de um estudo qualitativo realizado com enfermeiras atuantes em serviços dos três níveis de atenção à saúde de uma grande cidade do Nordeste brasileiro. Para nortear essa pesquisa, foi utilizado o paradigma da integralidade do cuidado, o qual se baseia em ações articuladas de prevenção, promoção e recuperação de agravos, considerando as singularidades dos sujeitos ${ }^{(9)}$.

A pesquisa foi realizada em três unidades de Atenção Primária à Saúde, três UPAs e dois hospitais da rede, todos pertencentes ao mesmo território. A aproximação com as participantes se deu a partir da autorização dos gestores responsáveis e posterior inserção dos pesquisadores nos referidos campos. Após isso, foram convidadas a participar da pesquisa enfermeiras com pelo menos um ano de atuação no serviço, excluindo aquelas que estavam afastadas. As entrevistas foram realizadas individualmente, em sala reservada, pelo pesquisador principal com o apoio de três bolsistas de iniciação científica, previamente treinados. Ressalta-se que os entrevistados não estavam em horário de serviço, não havendo alteração da dinâmica de trabalho.

A coleta de dados ocorreu entre setembro e novembro de 2018, por meio de entrevista semiestruturada guiada por um roteiro criado pelos pesquisadores e adaptado com base nos critérios censitários do $\mathrm{IBGE}^{(10)}$ (raça/cor autodeclarada, idade, gênero, orientação sexual, estado civil, formação acadêmica e tempo de atuação no mercado de trabalho), além da questão orientadora: Em sua prática assistencial, como você reconhece e conduz os casos de violência conjugal? As entrevistas foram gravadas e tiveram duração média de 45 minutos. O fechamento amostral ocorreu por meio da saturação teórica, compreendida como a suspensão de novos participantes quando os dados obtidos se apresentam com redundância ou repetição(11).

Todo o conteúdo gravado foi transcrito e, em seguida, submetido à avaliação pelas entrevistadas, a fim de garantir a representatividade das falas e posterior início do processo analítico, conforme recomendação do Consolidated criteria for reporting qualitative research (COREQ).

Os dados coletados foram sistematizados com o auxílio do software NVIVO11®, validado por um pesquisador com experiência no uso de softwares e, em seguida, organizados em ideias centrais e discursos síntese, por meio do Discurso do Sujeito Coletivo (DSC). A fidedignidade do uso deste método foi garantida por meio da adoção das etapas de transcrição das falas e análise do material verbal, extraindo-se as figuras metodológicas: Ideia Central - IC e suas Expressões-Chave - ECH; a partir da IC e ECH, compuseram-se vários discursos síntese que foram os discursos coletivos ${ }^{(11)}$.

Os dados foram organizados em duas categorias analíticas: "Reconhecimento dos casos de violência conjugal" e "Condução dos casos de violência conjugal" nos três níveis de atenção: atenção básica, média complexidade e alta complexidade. 1.673.856.

O estudo foi submetido para apreciação ética e aprovado sob parecer número

RESULTADOS

Participaram do estudo 47 enfermeiras, sendo 45 (95,7\%) do sexo feminino e $2(4,2 \%)$ do sexo masculino. Considerando que a maioria dos participantes foram mulheres, nesse estudo será utilizada a forma feminina para se referir ao conjunto de participantes. No que tange ao nível de atenção à saúde, 18 (38,3\%) participantes eram da Atenção Primária, 
$15(31,9 \%)$ das UPAS e 14 (29,8\%) dos hospitais. No que se refere à cor, 39 (82,9\%) se autodeclararam brancas. A faixa etária variou entre 20 e 51 anos e a média de atuação no mercado de trabalho foi de seis a 10 anos.

O discurso das enfermeiras atuantes na Atenção Primária revelou que o reconhecimento dos casos de violência perpassa pela observação por parte do agente comunitário de saúde, bem como pela identificação de sinais e sintomas durante a prática assistencial, o que é favorecido pela criação de vínculo e pela escuta qualificada. A partir da identificação, as enfermeiras realizam condutas iniciais como administração de medicamentos e curativos, além de compartilhar o caso com as equipes do Núcleo de Apoio à Saúde da Família (NASF) e, quando não conseguem dar resolutividade, encaminham para os demais níveis de atenção.

$\mathrm{Na}$ atenção secundária, as profissionais fazem a identificação já na triagem, por meio da observação e identificação das marcas corporais e comportamentos que, segundo elas, são característicos de mulheres que sofreram violência. No tocante às condutas, as enfermeiras utilizam protocolos assistenciais para o manejo clínico dos casos, além de cuidar das lesões físicas.

Já no contexto hospitalar, o reconhecimento das situações de violência ocorre pela observação do agravo que levou a mulher à internação, a exemplo de ferimentos por arma de fogo, contundentes e queimaduras graves. As condutas adotadas dependem da gravidade do caso, sendo focadas, principalmente, na resolução dos problemas corporais e, quando percebido, de ordem psicológica.

Os discursos coletivos serão apresentados no Quadro 1, refletindo o reconhecimento e a condução dos casos de violência conjugal nos níveis de atenção à saúde.

Quadro 1 - Discurso coletivo de enfermeiras acerca do conhecimento e condução dos casos de violência nos níveis de atenção. Salvador, BA, Brasil, 2020 (continua)

\begin{tabular}{|c|c|c|}
\hline $\begin{array}{l}\text { Níveis de } \\
\text { atenção }\end{array}$ & Reconhecimento da violência conjugal & Condução dos Casos \\
\hline $\begin{array}{l}\text { Atenção } \\
\text { Básica }\end{array}$ & $\begin{array}{l}\text { Na maioria das vezes, são os Agentes } \\
\text { Comunitários de Saúde que trazem para as } \\
\text { reuniões de equipe as situações de violência } \\
\text { conjugal que ocorrem na comunidade } \\
\text { [...] Quando essas mulheres procuram } \\
\text { atendimento, eu as acolho e escuto. É nesse } \\
\text { momento que eu percebo as demandas } \\
\text { não só físicas, mas também de ordem } \\
\text { psicológicas [...]Antes, a usuária vinha até } \\
\text { a unidade básica com um sorriso no rosto, } \\
\text { depois percebemos em seu semblante } \\
\text { uma tristeza profunda. Durante a realização } \\
\text { das consultas de enfermagem, eu também } \\
\text { percebo vestígios de violência física e sexual } \\
\text { [...] Existem sinais que são clássicos, como } \\
\text { os hematomas, arranhões e manchas [....] } \\
\text { Algumas delas relatam ausência de libido, } \\
\text { todavia, durante a realização do exame } \\
\text { físico da região perianal percebo lacerações } \\
\text { vaginal elou anal. (DSC, Enfermeiras que } \\
\text { atuavam na Atenção Básica à Saúde - USF, } \\
\text { UBS, CAPS) }\end{array}$ & $\begin{array}{l}\text { Não tenho um protocolo de atuação } \\
\text { para situações de violência contra a } \\
\text { mulher. Diante do atendimento de uma } \\
\text { vítima, eu presto a assistência necessária, } \\
\text { tal como curativos e administração } \\
\text { de medicamentos. Casos de maior } \\
\text { complexidade nós compartilhamos com } \\
\text { o NASF e, quando não temos condições } \\
\text { de manejar o caso, encaminhamos para } \\
\text { outros serviços [... (DSC, Enfermeiras que } \\
\text { atuavam na Atenção Básica à Saúde - } \\
\text { USF, UBS, CAPS) }\end{array}$ \\
\hline
\end{tabular}




\begin{tabular}{|c|c|c|}
\hline $\begin{array}{l}\text { Média } \\
\text { complexidade }\end{array}$ & $\begin{array}{l}\text { Logo na triagem, nós já percebemos algo } \\
\text { diferente [...] Na maioria das vezes, a mulher } \\
\text { que sofre violência apresenta lesões bem } \\
\text { características como hematomas no rosto, } \\
\text { braços e pernas [...] Às vezes elas chegam } \\
\text { acompanhadas por outra mulher, sendo a } \\
\text { mãe, irmã ou uma amiga. Em outras vem } \\
\text { acompanhada de uma viatura policial [...] } \\
\text { isso já nos deixava em alerta. Durante a } \\
\text { anamnese, as vítimas sempre ficam de } \\
\text { cabeça baixa, choravam bastante e não } \\
\text { conseguiam nos passar as informações. } \\
\text { Geralmente eu faço as perguntas a sual } \\
\text { seu acompanhante, que me relatava o } \\
\text { ocorrido. [...] Quando se trata de uma } \\
\text { situação de violência sexual, ela chegava } \\
\text { chorando muito, colocando a mão sobre a } \\
\text { região genital. Se quem estava de plantão } \\
\text { era um enfermeiro do sexo masculino, elas } \\
\text { não queriam ser atendidas por ele. (DSC, } \\
\text { enfermeiras que atuavam em unidades } \\
\text { de Pronto Atendimento, Ambulatórios } \\
\text { (Policlínicas) e Serviço de Atendimento } \\
\text { Móvel de Urgência - SAMU) }\end{array}$ & $\begin{array}{l}\text { Diante das situações de violência física, } \\
\text { nós nos restringimos aos cuidados } \\
\text { físicos, a exemplo da administração } \\
\text { de medicamentos, avaliação dos sinais } \\
\text { vitais e, diante de uma lesão de maior } \\
\text { gravidade, realizamos curativos ou } \\
\text { auxiliamos o médico na sutura [...] Nos } \\
\text { casos de violência sexual, seguíamos um } \\
\text { fluxo de atendimento. Se o ato tivesse } \\
\text { ocorrido em até } 72 \text { hs, nós acolhemos, } \\
\text { realizamos concepção de emergência, } \\
\text { conduzíamos para a realização de } \\
\text { exames laboratoriais, profilaxia de HIV } \\
\text { e IST e para exame pericial. Se essa } \\
\text { ocorresse após } 72 \text { horas, nós acolhemos } \\
\text { e encaminhamos para a realização de } \\
\text { exames laboratoriais. Em ambos os } \\
\text { casos, fazemos a notificação compulsória } \\
\text { da violência e encaminhávamos para } \\
\text { a assistência social e psicóloga. (DSC, } \\
\text { enfermeiras que atuavam em unidades } \\
\text { de Pronto Atendimento, Ambulatórios } \\
\text { (Policlínicas) e Serviço de Atendimento } \\
\text { Móvel de Urgência - SAMU) }\end{array}$ \\
\hline $\begin{array}{l}\text { Alta } \\
\text { complexidade }\end{array}$ & $\begin{array}{l}\text { Aqui no hospital, nós recebemos situações } \\
\text { extremas de violência física contra as } \\
\text { mulheres, a exemplo de ferimentos por } \\
\text { arma branca e de fogo que requerem } \\
\text { intervenções cirúrgicas. [...] Quando a } \\
\text { mulher chega com sinais de queimadura } \\
\text { em determinadas partes do corpo como } \\
\text { cabeça e membros, a gente já entende } \\
\text { que foi violência. (DSC, Enfermeiras que } \\
\text { atuavam na rede hospitalar - Unidades de } \\
\text { Obstetrícia, Emergência, Terapia Intensiva, } \\
\text { Vigilância Epidemiológica, Centro Cirúrgico } \\
\text { e Unidades de Internamento) }\end{array}$ & $\begin{array}{l}\text { Eu procuro abordar com respeito, sem } \\
\text { ser invasiva e sem fazer perguntas } \\
\text { constrangedoras, buscando estabelecer } \\
\text { vínculo com ela [...] Após isso, presto } \\
\text { a assistência que me cabe com base } \\
\text { naquilo que foi encontrado durante a } \\
\text { anamnese e o exame físico [...] Logo, } \\
\text { construo o plano de cuidado para que } \\
\text { essa mulher possa ser assistida da } \\
\text { melhor forma possível, não só no campo } \\
\text { físico, mas também no psicológico. } \\
\text { (DSC, Enfermeiras que atuavam na rede } \\
\text { hospitalar - Unidades de Obstetrícia, } \\
\text { Emergência, Terapia Intensiva, Vigilância } \\
\text { Epidemiológica, Centro Cirúrgico e } \\
\text { Unidades de Internamento) }\end{array}$ \\
\hline
\end{tabular}

Fonte: Os autores (2020).

O discurso coletivo das enfermeiras atuantes na Atenção Primária revelou que os Agentes Comunitários de Saúde (ACS) exercem papel fundamental na identificação da violência, uma vez que, pela maior proximidade com o contexto territorial, conseguem perceber os casos na comunidade, os quais são compartilhados nas reuniões de equipe. Esses profissionais, em geral, residem nas áreas onde trabalham e atuam no acompanhamento da situação de saúde da população adscrita, o que viabiliza o reconhecimento do agravo ${ }^{(12)}$.

As enfermeiras revelaram ainda que, quando as mulheres chegam até a unidade de saúde da família, é realizado o acolhimento no sentido de estimular o compartilhamento das suas vivências. Para isso, é imprescindível que os profissionais de saúde apresentem 
habilidades de comunicação que perpassem pela empatia e sensibilidade ${ }^{(13-14)}$. Tais elementos são indispensáveis para a formação do vínculo, o qual proporciona maior abertura na relação entre profissional e usuárias.

Por meio do vínculo, é possível que as enfermeiras não apenas colham informações, como também reconheçam mudanças no comportamento da mulher. Sob esta perspectiva, estudos afirmam que as mulheres que estão em situação de violência apresentam sinais sugestivos do agravo, dentre eles: tristeza, apatia, irritabilidade e dificuldade de concentração(15-16). Ressalta-se que o vínculo é um dos aspectos fundamentais da integralidade do cuidado, o que perpassa pelo interesse das profissionais pela sua situação, com uma escuta qualificada, sigilosa e sem julgamentos ${ }^{(9)}$.

As narrativas demonstraram ainda que, através do exame físico, é possível identificar a presença de lesões, hematomas e até mesmo vestígios de violência sexual, a exemplo de lacerações na região perianal. A visibilidade da violência conjugal está associada, no imaginário coletivo, quase que exclusivamente à presença de marcas no corpo feminino, sendo essas consideradas uma expressão do poder e dominação do homem para com sua companheira ${ }^{(17)}$.

O reconhecimento da violência conjugal apenas pelas marcas físicas leva as enfermeiras à prestação do cuidado exclusivamente voltado ao tratamento das lesões, não sendo fornecido um cuidado holístico. Além disso, a falta de um protocolo assistencial, somada à falta de conhecimento, dificultam o direcionamento das ações, a tomada de decisões e os encaminhamentos ${ }^{(18)}$.

Frente às situações de difícil manejo, as enfermeiras da atenção primária contam com o suporte da equipe do Núcleo de Apoio à Saúde da Família (NASF), que oferta apoio matricial a partir da discussão e acompanhamento conjunto dos $\operatorname{casos}^{(19)}$. Essa atuação multiprofissional com foco nas singularidades de cada mulher é essencial para a resolutividade das práticas de cuidado(9).

As enfermeiras da atenção secundária, por sua vez, revelaram que, além das marcas físicas, outros sinais podem direcionar à suspeita da violência, dentre eles a postura corporal e a expressão facial. As falas evidenciaram que as mulheres se apresentaram de cabeça baixa, com expressão de choro e dificuldade para relatar a queixa. Corroborando, estudo sérvio apontou que mulheres que procuraram os serviços de saúde da atenção especializada por causas violentas apresentaram sintomas comportamentais, tais como tristeza profunda, sinais de depressão e ideação suicida ${ }^{(15)}$.

Em situações de abuso sexual, outros comportamentos foram observados pelas enfermeiras, a exemplo do posicionamento da mão sob a genitália e a recusa do atendimento por profissionais do sexo masculino. Essas atitudes também foram evidenciadas em estudo brasileiro, o qual aponta manifestações corporais inconscientes como mecanismo de defesa(20), e em estudo realizado nos Estados Unidos, que demonstra a tendência de mulheres vítimas de abuso sexual se negarem a serem atendidas por homens ${ }^{(21)}$. Compreende-se assim que esses sinais comportamentais podem servir de alerta para a identificação da violência durante os atendimentos.

Outra característica constatada pelas enfermeiras foi que as mulheres geralmente chegam à unidade de saúde acompanhadas, seja por outra mulher ou por um policial. O fato de as vítimas, em sua maioria, buscarem atendimento acompanhadas por outras mulheres demonstra a relevância do suporte social para o encorajamento feminino, não apenas para a busca de ajuda médica, como também para a solicitação de apoio legal(22). Destaca-se, contudo, que, ao tempo que a presença policial pode representar segurança e proteção, por outro lado, também pode se manifestar como uma forma de revitimização da mulher, já que favorece a sua exposição perante aos demais pacientes ${ }^{(21)}$. Considera-se ainda a masculinidade hegemônica imbricada na formação dos homens, o que pode direcionar a conduta policial de julgamento da figura feminina e subestimaçã́o das queixas ${ }^{(23)}$. 
Ainda sob a perspectiva da revitimização, é necessário refletir sobre a invisibilidade do discurso feminino, já que as enfermeiras pesquisadas relataram direcionar as interpelações aos acompanhantes. Estudo brasileiro realizado com profissionais de saúde revela a invisibilização das mulheres em situação de violência no momento do atendimento, pela "rotulação" de algumas mulheres de poliqueixosas e os preconceitos oriundos do agravo, associados a concepções de culpabilização da mulher pela violência sofrida, exprimindo uma percepção sexista ${ }^{(24)}$. Considera-se que, mesmo que a instabilidade emocional dificulte a exposição das queixas, é importante que o profissional de saúde esteja apto a fornecer condições para que a mulher possa se expressar ${ }^{(25)}$.

No que se refere às condutas adotadas pelos profissionais no nível secundário de atenção à saúde, observa-se que estas permearam o cuidado com as lesões físicas, administração de medicamentos, realização de exames laboratoriais, notificação compulsória e encaminhamentos. Foi possível verificar uma maior dificuldade de colocar em prática o princípio da integralidade do cuidado, considerando que as práticas assistenciais se pautaram no modelo curativista, centrado na lógica dos atendimentos rápidos ${ }^{(9)}$.

No tocante à atenção terciária, os discursos demonstraram o atendimento de mulheres com repercussões físicas de maior gravidade em decorrência da violência de cunho físico. Levantamentos realizados no Brasil evidenciam que situações como queimaduras, fraturas, lesões por arma de fogo e arma branca são as principais implicações que levam ao internamento hospitalar, podendo deixar marcas permanentes na vida da mulher ${ }^{(26)}$.

Para as profissionais atuantes no serviço hospitalar, a aparência de marcas na cabeça e nos membros é um marcador que sinaliza que aquela mulher foi vítima de violência conjugal. Isso é ratificado em estudo brasileiro quando revela que a face, cabeça, pescoço e membros inferiores são os locais mais afetados nas agressões perpetradas por homens autores de violência ${ }^{(27)}$. Durante a ocorrência da violência, as mulheres são agredidas, na maioria das vezes, nos membros superiores, por tentarem, com esta parte do corpo, uma autodefesa, principalmente, de bofetadas e socos direcionados ao rosto. Vale destacar que agressão direcionada ao rosto é percebida como demonstração social da força e dominação masculina em relação a sua cônjuge, considerando que esta região do corpo é considerada um lócus corporal privilegiado e de alto valor simbólico ${ }^{(27)}$.

O discurso revelou ainda a realização de um plano de cuidados pautado nas necessidades das mulheres em situação de violência. Apesar de não ter havido um reconhecimento do uso da Sistematização da Assistência de Enfermagem, apontou-se a execução de algumas etapas do processo, a exemplo do histórico de enfermagem por meio da anamnese e exame físico, planejamento e implementação do plano de cuidados.

É necessária uma articulação dos três níveis de atenção, de forma a organizar as práticas assistenciais à mulher em situação de violência considerando fatores de ordem histórica, política, socioeconômica e individual, para a integralidade do cuidado( ${ }^{(9)}$. Embora a investigação limite-se por não apresentar uma relação integrada entre a prática da assistência nos três níveis de atenção à saúde de mulher em situação de violência, o estudo avança ao revelar, de forma didática, como se dá o reconhecimento e condução dos casos de violência entre os profissionais. Urge que a gestão priorize estratégias de cuidado direcionadas à capacitação profissional para o cuidado às mulheres que vivenciam a violência conjugal, em todos os níveis de atenção, contemplando as suas demandas e especificidades de maneira integral e humanizada.

\section{CONCLUSÃO}

O estudo descreve a prática assistencial da enfermeira frente aos casos de violência conjugal nos três níveis de atenção à saúde, que perpassam pela identificação e intervenção 
diante do agravo. Em que pese a identificação dos casos, a pesquisa despontou para a necessidade de investigação do cotidiano conjugal, estabelecimento de vínculo com a usuária e a articulação com outros profissionais, a exemplo dos ACS. O estudo destaca ainda a necessidade de escuta qualificada e articulação entre os profissionais que compõem a equipe mínima e o NASF, considerando maior relação de proximidade com as mulheres.

A pesquisa oferece subsídios para nortear o cuidado às mulheres em situação de violência conjugal nos três níveis de complexidade. Sugere-se que as estratégias evidenciadas, a exemplo da identificação dos casos com a criação de vínculo com as usuárias e a articulação com outros profissionais da rede de atenção à mulher em situação de violência, sejam integradas aos fluxos construídos, de modo a facilitar a integração entre os níveis de atenção e até mesmo auxiliar no processo de capacitação profissional para um cuidado integral holístico e humanizado. Considerando que a enfermagem é a profissão com maior contato e proximidade no cuidado prestado a essas mulheres, acredita-se que o uso das estratégias apontadas neste estudo poderá servir como eixo norteador de um cuidado integral, holístico, humanizado e com foco na prevenção do agravo.

\title{
REFERÊNCIAS
}

\author{
1. Paim JS. O que é o SUS. Rio de Janeiro: Editora Fiocruz; 2009.
}

2. World Health Organization (WHO). Violence against women [Internet]. 2017 [acesso em 28 abr 2020]. Disponível em: https://www.who.int/news-room/fact-sheets/detail/violence-against-women.

3. Fórum Brasileiro de Segurança Pública. Datafolha. Visível e invisível: a vitimização de mulheres no Brasil. 2. ed. [Internet]. 2019 [acesso em 28 abr 2020]. Disponível em: https://www12.senado.leg.br/ institucional/procuradoria/proc-publicacoes/relatorio-vitimizacao-de-mulheres-no-brasil-2deg-edicao.

4. Brasil. Lei n. 11.340, de 7 de agosto de 2006. Cria mecanismos para coibir a violência doméstica e familiar contra a mulher, nos termos do $\S 8^{\circ}$ do art. 226 da Constituição Federal, da Convenção sobre a Eliminação de Todas as Formas de Discriminação contra as Mulheres e da Convenção Interamericana para Prevenir, Punir e Erradicar a Violência contra a Mulher; dispõe sobre a criação dos Juizados de Violência Doméstica e Familiar contra a Mulher; altera o Código de Processo Penal, o Código Penal e a Lei de Execução Penal; e dá outras providências. Diário Oficial da República Federativa do Brasil, Brasília, 7 ago. 2006.

5. Tscholl JJ, Scribano PV. Intimate partner violence. In: A practical guide to the evaluation of child physical abuse and neglect. New York: Springer International Publishing; 2019 [acesso em 20 abr 2020]. p. 355-73. Disponível em: http://doi.org/10.1007/978-3-030-00635-8_11.

6. Waiselfisz JJ. Mapa da violência 2015: homicídio de mulheres no Brasil - Vol. 1. [Internet]. Brasília: Flacso Brasil; 2015 [acesso em 29 set 2017]; 83 p. Disponível em: http://www.onumulheres.org.br/wpcontent/uploads/2016/04/MapaViolencia 2015 mulheres.pdf.

7. Souza TMC, Rezende FF. Violência contra mulher: concepções e práticas de profissionais de serviços públicos. Est. Inter Psicol. [Internet]. 2018 [acesso em 28 abr 2020]; 9(2). Disponível em: http://pepsic. bvsalud.org/scielo.php?script=sci arttext\&pid=S2236-64072018000200003\&lng=pt\&tlng=pt.

8. Lawrenz P, Macedo DM, Hohendorff J von, Freitas CPP de, Foschiera LN, Habigzang LF. Violence against women: notifications of health professionals in Rio Grande do Sul. Psicol Teor e Pesqui. [Internet]. 2019 [acesso em 22 abr 2020]; 34(e34428). Disponível em: http://doi.org/10.1590/0102.3772e34428.

9. Alves LR. A dicotomia do princípio da integralidade do SUS. Cad. Ibero-Amer. Dir. Sanit. [Internet]. 2017 [acesso em 28 jul 2020]; 6(1). Disponível em: http://dx.doi.org/10.17566/ciads.v6i1.338A.

10. Melo MP de, Lessa SE do C. Healthcare Politics, Neoliberal Project and the Growth of the Third 
Sector: an analysis of the FASFIL from de healthcare field in Censo IBGE 2005. Textos \& Contextos (Porto Alegre) [Internet]. 2017 [acesso em 28 jul 2020]; 16(1). Disponível em: https://revistaseletronicas.pucrs.br/ ojs/index.php/fass/article/view/24602/0.

11. Lefevre F, Lefevre AMC. Discourse of the collective subject: social representations and communication interventions. Texto contexto-enferm. [Internet] 2014 [acesso em 20 abr 2020]; 23(2). Disponível em: http://doi.org/10.1590/0104-07072014000000014.

12. Tonsing J, Barn R. Intimate partner violence in South Asian communities: Exploring the notion of 'shame' to promote understandings of migrant women's experiences. Int Soc Work. [Internet]. 2016 [acesso em 15 abr 2020]; 60(3). Disponível em: http://doi.org/10.1177/0020872816655868.

13. Othman S, Yuen CW, Zain MN, Samad AA. Exploring intimate partner violence among women attending malaysian primary care clinics. J Interpers Violence. [Internet]. 2019 [acesso em 25 abr 2020]. Disponível em: http://doi.org/10.1177/0886260519839426.

14. Machado ME de S, Rodrigues LS de A, Sorte ETB, Silva JM da, Silva D de O, Oliveira JF de. Percepção de profissionais de saúde sobre violência contra a mulher: estudo descritivo. Online Braz J Nurs. (Online). [Internet]. 2017 [acesso em 12 abr 2020]; 16(2). Disponível em: https://pesquisa.bvsalud. org/portal/resource/pt/biblio-877011.

15. Knezevic S, Gajovic I, Marinkovic A. Barriers to health care professionals in detecting more domestic violence. Opsta Med. [Internet]. 2017 [acesso em 20 abr 2020]; 23(3-4). Disponível em: http://doi. org/10.5937/opmed1703067K.

16. Holanda ER de, Holanda VR de, Vasconcelos MS de, Souza VP de, Galvão MTG. Fatores associados à violência contra as mulheres na atenção primária de saúde. Rev. bras. promoç. saúde. [Internet]. 2018 [acesso em 20 abr 2020]; 31(1). Disponível em: http://doi.org/10.5020/18061230.2018.6580.

17. Connell RW, Messerschmidt JW. Masculinidade hegemônica: repensando o conceito. Rev. Estud. Fem. [Internet]. 2013 [acesso em 20 abr 2020]; 21(1). Disponível em: http://doi.org/10.1590/S0104026X2013000100014.

18. Tozija F. Women's safety and gender-based violence in the republic of North Macedonia. Front Public Heal. [Internet] 2020 [acesso em 20 abr 2020]; 8. Disponível em: https://doi.org/10.3389/ fpubh.2020.00033.

19. Torralbas-Fernández A, Calcerrada-Gutiérrez M. Using primary care to address violence against women in intimate partner relationships: professional training needs. MEDICC Rev [Internet]. 2016 [acesso em 28 abr 2020];18(4). Disponível em: http://www.ncbi.nlm.nih.gov/pubmed/27829653.

20. Soto CAA. Discurso de los lectores de médios digitales ante la violencia policial contra la mujer: ¿otra forma de violencia virtual? Estudios sobre el mensaje periodistico [Internet]. 2018 [acesso em $20 \mathrm{abr}$ 2020]; 24(2). Disponível em: https://revistas.ucm.es/index.php/ESMP/article/view/62200.

21. Pividori C, Degani P. Reflecting on criminalizing male violence against women under human rights and human security discourses: a feminist legal and policy analysis. Glob Jurist [Internet]. 2018 [acesso em 20 abr 2020]; 19(1). Disponível em: http://doi.org/10.1515/gj-2017-0028.

22. Castro RJ, Cerellino LP, Rivera R. Risk factors of violence against women in Peru. J Fam Violence. [Internet]. 2017 [acesso em 20 abr 2020]; 32. Disponível em: http://doi.org/10.1007/s10896-017-9929-0.

23. Silva NNF, Leal SMC, Trentin D, Vargas MA de O, Vargas CP, Vieira LB. Atuação dos enfermeiros da atenção básica a mulheres em situação de violência. Enferm em Foco. [Internet]. 2017 [acesso em 20 abr 2020]; 8(3). Disponível em: http://doi.org/10.21675/2357-707X.2017.v8.n3.1290.

24. Martínez Al, Sánchez PM. Invisibilidad de la violencia de género en mujeres con diversidad funcional. Int J Dev Educ Psychol Rev INFAD Psicol. [Internet]. 2017 [acesso em 20 abr 2020]; 4(1). Disponível em: http://doi.org/10.17060/ijodaep.2017.n1.v4.1042. 
demográficos ou sociodemográficos de mulheres em situação de violência conjugal: estudo descritivo. Online Brazilian J Nurs. [Internet]. 2016 [acesso em 20 abr 2020]; 15(3). Disponível em: http://doi. org/10.17665/1676-4285.20165380.

26. Estrela FM, Gomes NP, Lírio JG dos S, Silva AF da, Mota RS, Pereira A, et al. Expressions and repercussions of conjugal violence: processes of women in a probate court. Rev enferm UFPE online. [Internet]. 2018 [acesso em 00 mês abreviado 0000]; 12(9). Disponível em: http://doi.org/10.5205/19818963-v12i9a231013p2418-2427-2018.

27. Dourado S de M, Noronha CV. A face marcada: as múltiplas implicações da vitimização feminina nas relações amorosas. Physis [Internet]. 2014 [acesso em 20 abr 2020]; 24(2). Disponível em: http://doi. org/10.1590/S0103-73312014000200016.

COMO REFERENCIAR ESTE ARTIGO:

Sousa AR de, Estrela FM, Silva AF da, Magalhães JRF, Oliveira MA da S, Loureiro AKN da S, et al. Violência conjugal e prática assistencial por níveis de atenção à saúde: discurso de enfermeiras. Cogitare enferm. [Internet]. 2021 [acesso em "colocar data de acesso, dia, mês abreviado e ano"]; 26. Disponível em: http://dx.doi.org/10.5380/ ce.v26i0.74083.

Recebido em: 27/05/2020

Aprovado em: 03/08/2020

Editora associada: Tatiane Herreira Trigueiro

Autor Correspondente:

Fernanda Matheus Estrela

Universidade Estadual de Feira de Santana - Feira de Santana, BA, Brasil

E-mail: nanmatheus@yahoo.com.br

Contribuição dos autores:

Contribuições substanciais para a concepção ou desenho do estudo; ou a aquisição, análise ou interpretação de dados do estudo - ARS, FME, AFS, JRFM, MASO, AKNSL, VRG

Elaboração e revisão crítica do conteúdo intelectual do estudo - ARS, FME, AFS, JRFM, MASO, AKNSL, VRG Aprovação da versão final do estudo a ser publicado - ARS, FME, AFS, JRFM, MASO, AKNSL, VRG

Copyright () 2021 Este é um artigo em acesso aberto distribuído nos termos da Licença Creative Commons Atribuição, que permite o uso irrestrito, a distribuição e reprodução em qualquer meio desde que o artigo original seja devidamente citado. 